\title{
Stability of cascaded Takagi-Sugeno fuzzy systems*
}

\author{
Zs. Lendek, R. Babuška, and B. De Schutter \\ If you want to cite this report, please use the following reference instead: \\ Zs. Lendek, R. Babuška, and B. De Schutter, "Stability of cascaded Takagi-Sugeno \\ fuzzy systems," Proceedings of the 2007 IEEE International Conference on Fuzzy \\ Systems (FUZZ-IEEE 2007), London, UK, pp. 505-510, July 2007.
}

Delft Center for Systems and Control

Delft University of Technology

Mekelweg 2, 2628 CD Delft

The Netherlands

phone: +31-15-278.24.73 (secretary)

URL: https://www.dcsc.tudelft.nl

*This report can also be downloaded via https://pub.deschutter.info/abs/07_012.html 


\title{
Stability of Cascaded Takagi-Sugeno Fuzzy Systems
}

\author{
Zs. Lendek $\quad$ R. Babuška $\quad$ B. De Schutter
}

\begin{abstract}
A large class of nonlinear systems can be well approximated by Takagi-Sugeno (TS) fuzzy models, with local models often chosen linear or affine. It is well-known that the stability of these local models does not ensure the stability of the overall fuzzy system. Therefore, several stability conditions have been developed for TS fuzzy systems. We study a special class of nonlinear dynamic systems, that can be decomposed into cascaded subsystems. These subsystems are represented as TS fuzzy models. We analyze the stability of the overall TS system based on the stability of the subsystems. For a general nonlinear, cascaded system, global asymptotic stability of the individual subsystems is not sufficient for the stability of the cascade. However, for the case of TS fuzzy systems, we prove that the stability of the subsystems implies the stability of the overall system. The main benefit of this approach is that it relaxes the conditions imposed when the system is globally analyzed, therefore solving some of the feasibility problems. Another benefit is, that by using this approach, the dimension of the associated linear matrix inequality (LMI) problem can be reduced. Applications of such cascaded systems include multiagent systems, distributed process control and hierarchical large-scale systems.
\end{abstract}

\section{INTRODUCTION}

Dynamic systems are often modeled in the state-space framework, using a state-transition model, which describes the evolution of states over time and a measurement (sensor) model, which relates the measurements to the states.

Traditionally, the class of linear, time-invariant systems have dominated control theory. The linearity and timeinvariance make these types of systems easy to analyze. The disadvantage is that such models usually fail to describe nonlinear systems globally. An accurate approximation of a nonlinear system can only be expected in the vicinity of an equilibrium point or trajectory.

A large class of nonlinear systems can be well approximated by TS fuzzy models [1], which in theory can approximate a general nonlinear system to an arbitrary accuracy [2].

The TS fuzzy model consists of a fuzzy rule base. The antecedents of the rules partition a given subspace of the model variables into fuzzy regions. The consequent of each rule is usually a linear model, valid locally in the region defined by the corresponding antecedent.

Although the local models are often chosen to be linear or affine, the stability of these models does not ensure the stability of the fuzzy model. Therefore, several stability conditions have been developed for TS fuzzy systems, most of them relying on the feasibility of an associated system of linear matrix inequalities (LMI) [3]-[6]. However, the

The authors are with the Delft Center for Systems and Control, Delft University of Technology, Mekelweg 2, 2628 CD Delft, The Netherlands (email: zs.lendek@tudelft.nl, r.babuska@tudelft.nl).

B. De Schutter is also with the Marine and Transport Technology Department of the Delft University of Technology (email: b@ deschutter.info) complexity of the system grows exponentially with the number of antecedents and the stability analysis problem eventually becomes intractable for a large number of rules.

An important class of nonlinear dynamic systems can be represented as cascaded subsystems. In several cases, conclusions referring to the overall system can be drawn based on the study of the individual subsystems. E. g., for linear systems, the stability of the individual subsystems imply the stability of the cascaded system [7]. This property, however, in general does not hold for nonlinear or timevarying systems. Even global asymptotic stability of the individual subsystems does not necessarily imply stability of the cascade.

In the literature, the stability of several types of cascaded systems has been studied. The main motivation came from the linear-nonlinear cascade [8], resulting from input-output linearization. Conditions to ensure the overall stability of more general cascades, in which all subsystems are nonlinear, were derived in [7], [9], [10].

We study a special class of systems, represented as TS fuzzy systems, which can be decomposed into cascaded subsystems, and analyze the stability of the whole system based on the stability of the subsystems. This class of systems is very important, as many systems are naturally distributed (e.g., multi-agent systems) or cascaded (e.g., hierarchical large-scale systems). Others may be represented as cascaded subsystems, which are less complex than the original system. The main benefit of this approach is, that it relaxes the conditions imposed by analyzing the global system. A global analysis of the system may lead to infeasible LMI problems, even if the analyzed system is stable. In this paper, we propose more relaxed stability conditions, which may render the associated LMI problem feasible. Moreover, for some applications, the dimension of the associated LMI system is greatly reduced. The results presented can also be extended to observer design for the class of cascaded systems.

The structure of the paper is as follows. Section 2 introduces the proposed cascaded setting for nonlinear systems and presents stability conditions for cascaded systems and for TS fuzzy models. The proposed stability conditions for cascaded fuzzy systems are presented in Section 3. Examples are given in Section 4. Finally, Section 5 concludes the paper. 


\section{Stability of CAscaded Dynamic Systems}

\section{A. Preliminaries}

Consider the following $n$th order, general nonlinear system with $m$ outputs:

$$
\begin{array}{cc}
\dot{x}_{1}=f_{1}(\boldsymbol{x}, \boldsymbol{u}) & y_{1}=h_{1}(\boldsymbol{x}, \boldsymbol{u}) \\
\dot{x}_{2}=f_{2}(\boldsymbol{x}, \boldsymbol{u}) & y_{2}=h_{2}(\boldsymbol{x}, \boldsymbol{u}) \\
\vdots & \vdots \\
\dot{x}_{n}=f_{n}(\boldsymbol{x}, \boldsymbol{u}) & y_{m}=h_{m}(\boldsymbol{x}, \boldsymbol{u})
\end{array}
$$

Assume that this system (both the states and the outputs) can be partitioned into several subsystems. For the ease of notation, two subsystems are considered here (without loss of generality):

$$
\begin{aligned}
& \dot{\boldsymbol{x}}_{1}=\boldsymbol{f}_{1}\left(\boldsymbol{x}_{1}, \boldsymbol{u}\right) \\
& \boldsymbol{y}_{1}=\boldsymbol{h}_{1}\left(\boldsymbol{x}_{1}, \boldsymbol{u}\right)
\end{aligned}
$$

and

$$
\begin{aligned}
& \dot{\boldsymbol{x}}_{2}=\boldsymbol{f}_{2}\left(\boldsymbol{x}_{1}, \boldsymbol{x}_{2}, \boldsymbol{u}\right) \\
& \boldsymbol{y}_{2}=\boldsymbol{h}_{2}\left(\boldsymbol{x}_{1}, \boldsymbol{x}_{2}, \boldsymbol{u}\right)
\end{aligned}
$$

with $\boldsymbol{x}=\boldsymbol{x}_{1} \cup \boldsymbol{x}_{2}$ and $\boldsymbol{x}_{1} \cap \boldsymbol{x}_{2}=\emptyset$.

In general, such a partition of the model may not necessarily exist, and, if it exists, it might not be unique. Since stability is independent of the measurement models $\boldsymbol{h}_{1}$ and $\boldsymbol{h}_{2}$, in the sequel, the models $\boldsymbol{h}_{1}$ and $\boldsymbol{h}_{2}$ are not considered. Then, for two subsystems, the cascaded structure is depicted in Figure 1.

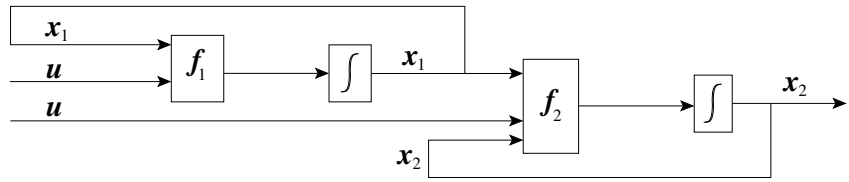

Fig. 1. Cascaded subsystems.

\section{B. Stability of Cascaded Systems}

It is well-known that the cascade of stable linear systems is stable, since the eigenvalues of the joint system are determined only by the eigenvalues of the individual subsystems [7]. Therefore, the stability of the subsystems implies the stability of the joint system. However, the same reasoning does not necessarily hold for nonlinear or timevarying systems. Even global asymptotic stability (GAS) of the individual subsystems does not necessarily imply the stability of the cascade.

In the literature, the stability of several special cases is studied. The main motivation comes from a linear-nonlinear cascade resulting from input-output linearization [8]. More general cascades in which both subsystems are nonlinear were studied and conditions to ensure the overall stability were presented in [7]. Several relevant results are presented below.

Definition 1: A continuous function $\alpha: \mathcal{R}^{+} \rightarrow \mathcal{R}^{+}$ belongs to class $\mathcal{K}$ if it is strictly increasing and $\alpha(0)=0$.
If $\alpha(s) \rightarrow \infty$ when $s \rightarrow \infty$, then $\alpha$ is said to be of class $\mathcal{K}_{\infty}$

Consider the nonlinear, cascaded, autonomous system

$$
\begin{aligned}
& \dot{\boldsymbol{x}}_{1}=\boldsymbol{f}_{1}\left(\boldsymbol{x}_{1}\right) \\
& \dot{\boldsymbol{x}}_{2}=\boldsymbol{f}_{2}\left(\boldsymbol{x}_{1}, \boldsymbol{x}_{2}\right) .
\end{aligned}
$$

It has been shown in [11] that if

- the functions $f_{1}$ and $f_{2}$ are sufficiently smooth in their arguments,

- system (5) is input-to-state-stable with regard to the input $\boldsymbol{x}_{1}$, and

- $\operatorname{system~(4)~and~}$

$$
\dot{\boldsymbol{x}}_{2}=\boldsymbol{f}_{2}\left(0, \boldsymbol{x}_{2}\right)
$$

are GAS,

then the cascaded system (4)-(5) is GAS. An equivalent sufficient stability condition is presented in [10]: the cascaded system is GAS, if both subsystems are GAS and all solutions are bounded. The main difficulty with this approach is that in general, boundedness of all the solutions is not easy to determine and the conditions to ensure boundedness may be very conservative.

More relaxed sufficient stability conditions have been derived for systems of the form:

$$
\begin{aligned}
& \dot{\boldsymbol{x}}_{1}=\boldsymbol{f}_{1}\left(\boldsymbol{x}_{1}\right) \\
& \dot{\boldsymbol{x}}_{2}=\boldsymbol{f}_{2}\left(\boldsymbol{x}_{2}\right)+\boldsymbol{g}\left(\boldsymbol{x}_{1}, \boldsymbol{x}_{2}\right)
\end{aligned}
$$

assuming that the individual subsystems are GAS and, additionally, certain restrictions related to the continuity and/or slope, apply for the interconnection term $\boldsymbol{g}$ [8], [12], [13].

A theorem for the uniform GAS (UGAS) of the cascaded system (7) [7] is presented below.

\section{Assumptions:}

1) System (6) is UGAS.

2) There exist constants $c_{1}, c_{2}, \mu>0$ and a Lyapunov function $V\left(t, \boldsymbol{x}_{2}\right)$ for (6) such that $V: \mathcal{R}^{+} \times$ $\mathcal{R}^{n} \rightarrow \mathcal{R}^{+}$is positive definite, radially unbounded, $\dot{V}\left(t, \boldsymbol{x}_{2}\right) \leq 0$ and

$$
\begin{array}{ll}
\left\|\frac{\partial V}{\partial \boldsymbol{x}_{2}}\right\|\left\|\boldsymbol{x}_{2}\right\| \leq c_{1} V\left(t, \boldsymbol{x}_{2}\right) & \forall\left\|\boldsymbol{x}_{2}\right\|>\mu \\
\left\|\frac{\partial V}{\partial \boldsymbol{x}_{2}}\right\| \leq c_{2} & \forall\left\|\boldsymbol{x}_{2}\right\| \leq \mu
\end{array}
$$

3) There exist two continuous functions $\theta_{1}, \theta_{2}: \mathcal{R}^{+} \rightarrow$ $\mathcal{R}^{+}$such that $\boldsymbol{g}(\boldsymbol{x})$ satisfies:

$$
\|\boldsymbol{g}(\boldsymbol{x})\| \leq \theta_{1}\left(\left\|\boldsymbol{x}_{1}\right\|\right)+\theta_{2}\left(\left\|\boldsymbol{x}_{1}\right\|\right)\left\|\boldsymbol{x}_{2}\right\|
$$

4) There exists a class $\mathcal{K}$ function $\alpha(\cdot)$ so that for all $t_{0} \geq 0$, the trajectories of the system (4) satisfy

$$
\int_{t_{0}}^{\infty}\left\|\boldsymbol{x}_{1}\left(t ; t_{0}, \boldsymbol{x}_{1}\left(t_{0}\right)\right)\right\| d t \leq \alpha\left(\left\|\boldsymbol{x}_{1}\left(t_{0}\right)\right\|\right)
$$

Theorem 1: Let Assumption 1 hold and suppose that the trajectories of (4) are uniformly globally bounded. If, in addition, Assumptions 2-4 are satisfied, then the solutions of the 
system (7) are uniformly globally bounded. If furthermore, system (4) is UGAS, then so is the cascaded system (7).

Proposition 1: If in addition to the above assumptions systems (4) and (6) are exponentially stable, then the cascaded system (7) is also exponentially stable.

The proof of the Theorem 1 and the study of different cases of the interconnection term can be found in [7], [8]. Stabilizability conditions for such cascaded systems were derived in [13], [14].

\section{Stability of Fuzzy Systems}

Consider an autonomous fuzzy system expressed as:

$$
\dot{\boldsymbol{x}}=\sum_{i=1}^{m} w_{i}(\boldsymbol{z}) A_{i} \boldsymbol{x},
$$

where $A_{i}, i=1,2, \ldots m$ is the state matrix of the $i$ th local linear model, $w_{i}$ is its corresponding normalized membership function, and $z$ denotes the scheduling vector. System (11) can also be regarded as a linear parameter varying (LPV) system:

$$
\dot{\boldsymbol{x}}=A(\boldsymbol{z}) \boldsymbol{x}
$$

with $A(\boldsymbol{z})=\sum_{i=1}^{m} w_{i}(\boldsymbol{z}) A_{i}$.

For the system (11), several stability conditions were derived. Among them, a well-known and frequently used condition is formulated below [3].

Theorem 2: System (11) is exponentially stable if there exist $P=P^{T}>0$ so that $A_{i}^{T} P+P A_{i}^{T}<0$, for all $i=$ $1,2, \ldots, m$.

A similar condition is used if the fuzzy system is subject to vanishing disturbances (perturbations):

$$
\dot{\boldsymbol{x}}=\sum_{i=1}^{m} w_{i}(\boldsymbol{z}) A_{i} \boldsymbol{x}+D \boldsymbol{f}(t, \boldsymbol{x})
$$

where $D$ is a perturbation distribution matrix and $f$ is a vanishing disturbance, i.e., $\boldsymbol{f}(t, \boldsymbol{x}) \rightarrow 0$ when $t \rightarrow \infty$, and $\boldsymbol{f}$ is Lipschitz, i.e., there exists $\mu>0$ so that $\|\boldsymbol{f}(t, \boldsymbol{x})\| \leq \mu\|\boldsymbol{x}\|$, for all $t$ and $\boldsymbol{x}$. With these assumptions, a sufficient stability condition can be formalized by the following theorem.

Theorem 3: System (13) is exponentially stable if there exist matrices $P=P^{T}, Q=Q^{T}$, so that

$$
\begin{aligned}
& P>0 \quad Q>0 \\
& \mu \leq \frac{\lambda_{\min }(Q)}{\|P D\|_{2}} \\
& A_{i}^{T} P+P A_{i}<-2 Q \quad i=1,2, \ldots, m
\end{aligned}
$$

Several variants of the above theorem exist, together with algorithms to compute robustness measures [15]. However, these approaches are conservative, by disregarding the fact that the local models are valid only in a region of the state-space. For fuzzy systems, the membership functions often have a bounded support. Therefore, it is sufficient that $\boldsymbol{x}^{T}\left(A_{i}^{T} P+P A_{i}^{T}\right) \boldsymbol{x}<0$ only where $w_{i}(\boldsymbol{z})>0$. Stability conditions for the case when the support of each membership function is bounded were derived in [4].
Another approach, based on partitioning the state-space into operating and interpolation regimes, is described in [16]. Assuming that in (11), $z$ can be expressed as some function of $\boldsymbol{x}$, the system can be written as:

$$
\dot{\boldsymbol{x}}=\sum_{i \in K_{k}} w_{i}(\boldsymbol{x}) A_{i} \boldsymbol{x} \quad \boldsymbol{x} \in X_{k}
$$

where $K_{k}$ is the index set of the linear subsystems active in the region $X_{k}$.

Then, using a Lyapunov function of the form $V(\boldsymbol{x})=$ $\boldsymbol{x}^{T} P_{i} \boldsymbol{x}$ when $\boldsymbol{x} \in X_{i}$, the system (15) is stable, under the conditions expressed by the following theorem [16].

Theorem 4: System (15) is stable, if there exist matrices $P_{i}=P_{i}^{T}, H=H^{T}>0, F_{i}, i=1,2, \ldots,|K|$, so that:

$$
\begin{array}{lr}
P_{i}=F_{i}^{T} H F_{i} & \\
P_{i}>0 & \\
F_{i} \boldsymbol{x}=F_{j} \boldsymbol{x} & \forall \boldsymbol{x} \in X_{i} \cap X_{j} \\
A_{k}^{T} P_{i}+P_{i} A_{k}<0 & \forall k \in K_{i}
\end{array}
$$

For more relaxed conditions, and the computations of the corresponding matrices see [17], [18]. Similar conditions for the discrete-time case are described in [19].

Last, but not least, for a parameter-dependent Lyapunov function of the form:

$$
V(\boldsymbol{x})=\boldsymbol{x}^{T} P(\boldsymbol{z}) \boldsymbol{x}=\boldsymbol{x}^{T} \sum_{i=1}^{m} w_{i}(\boldsymbol{z}) P_{i} \boldsymbol{x}
$$

the stability conditions can be formulated as follows [15].

Theorem 5: The system (11) is stable if there exist $\alpha>0$, $\bar{P}=\bar{P}^{T}>0, P_{i}=P_{i}^{T}>0, \Delta_{i}=\Delta_{i}^{T}>0, Q=Q^{T}>0$ so that

$$
\begin{aligned}
& \bar{P}-\Delta_{i} \leq P_{i} \\
& \bar{P}+\Delta_{i} \geq P_{i} \\
& G_{i i} \leq-Q \\
& G_{i j}+G_{j i} \leq-Q \\
& \sum_{i=1}^{m} \Delta_{i}<\alpha Q
\end{aligned}
$$

where $G_{i j}=A_{i}^{T} P_{j}+P_{j} A_{i}, i, j=1,2, \ldots, m$.

Note that all the above conditions rely on the feasibility of the corresponding linear matrix inequality problem. Since efficient algorithms exist for solving LMIs, they can be easily verified. However, two shortcomings of the above theorems have to be mentioned: 1) the conditions are conservative and often lead to infeasible LMIs and 2) the number of LMIs can grow exponentially with the number of local models, depending on the support of the membership functions (in particular for Theorems 4-5).

\section{Stability of Cascaded FuZzy Systems}

Consider the case when the system matrices of the model (11) for each rule $i=1,2, \ldots, m$ can be written as:

$$
A_{i}=\left(\begin{array}{cc}
A_{1} & 0 \\
A_{21} & A_{2}
\end{array}\right)_{i}=\left(\begin{array}{cc}
A_{1 i} & 0 \\
A_{21 i} & A_{2 i}
\end{array}\right)
$$


i.e., the system can be expressed as the cascade of two fuzzy systems:

$$
\begin{aligned}
& \dot{\boldsymbol{x}}_{1}=\sum_{i=1}^{m} w_{1 i}\left(\boldsymbol{z}_{1}\right) A_{1 i} \boldsymbol{x}_{1} \\
& \dot{\boldsymbol{x}}_{2}=\sum_{i=1}^{m} w_{2 i}(\boldsymbol{z})\left(A_{21 i} \boldsymbol{x}_{1}+A_{2 i} \boldsymbol{x}_{2}\right)
\end{aligned}
$$

or, equivalently:

$$
\begin{aligned}
& \dot{\boldsymbol{x}}_{1}=A_{1}\left(\boldsymbol{z}_{1}\right) \boldsymbol{x}_{1} \\
& \dot{\boldsymbol{x}}_{2}=A_{21}(\boldsymbol{z}) \boldsymbol{x}_{1}+A_{2}(\boldsymbol{z}) \boldsymbol{x}_{2}
\end{aligned}
$$

with

$$
\begin{aligned}
& A_{1}\left(\boldsymbol{z}_{1}\right)=\sum_{i=1}^{m} w_{1 i}\left(\boldsymbol{z}_{1}\right) A_{1 i} \boldsymbol{x}_{1}, \\
& A_{21}(\boldsymbol{z})=\sum_{i=1}^{m} w_{2 i}(\boldsymbol{z}) A_{21 i} \boldsymbol{x}_{1},
\end{aligned}
$$

etc.

Below, we prove that if the subsystems

$$
\dot{\boldsymbol{x}}_{1}=A_{1}\left(\boldsymbol{z}_{1}\right) \boldsymbol{x}_{1}
$$

and

$$
\dot{\boldsymbol{x}}_{2}=A_{2}(\boldsymbol{z}) \boldsymbol{x}_{2}
$$

are stable, it is possible to apply Theorem 1 to fuzzy systems of the form (19), i.e.,

Theorem 6: If there exist two Lyapunov functions of the form $V_{1}\left(\boldsymbol{x}_{1}\right)=\boldsymbol{x}_{1}^{T} P_{1} \boldsymbol{x}_{1}$ and $V_{2}\left(\boldsymbol{x}_{2}\right)=\boldsymbol{x}_{2}^{T} P_{2} \boldsymbol{x}_{2}$ so that the subsystems (21) and (22) are UGAS, then the cascaded system (20) is also UGAS.

Proof: Consider two Lyapunov functions of the form $V_{1}\left(\boldsymbol{x}_{1}\right)=\boldsymbol{x}_{1}^{T} P_{1} \boldsymbol{x}_{1}$ and $V_{2}\left(\boldsymbol{x}_{2}\right)=\boldsymbol{x}_{2}^{T} P_{2} \boldsymbol{x}_{2}$ for the subsystems (21) and (22). Note that such Lyapunov functions satisfy Assumptions 1 and 4 and also ensure exponential stability. Furthermore,

- Assumption 2 is satisfied as: $\forall\left\|\boldsymbol{x}_{2}\right\|>\mu$,

$$
\begin{aligned}
\left\|\frac{\partial V_{2}}{\partial \boldsymbol{x}_{2}}\right\|\left\|\boldsymbol{x}_{2}\right\| & =2\left\|\boldsymbol{x}_{2}^{T}\right\|\left\|P_{2}\right\|\left\|\boldsymbol{x}_{2}\right\| \\
& \leq 2 \lambda_{\max }\left(P_{2}\right)\left\|\boldsymbol{x}_{2}\right\|^{2} \leq c_{1} V_{2}\left(\boldsymbol{x}_{2}\right)
\end{aligned}
$$

for any $c_{1} \geq \frac{2 \lambda_{\max }\left(P_{2}\right)}{\lambda_{\min }\left(P_{2}\right)}$. For the second condition of Assumption 2, we have $\forall\left\|\boldsymbol{x}_{2}\right\| \leq \mu$,

$$
\begin{aligned}
\left\|\frac{\partial V_{2}}{\partial \boldsymbol{x}_{2}}\right\| & =\left\|2 \boldsymbol{x}_{2}^{T} P_{2}\right\| \\
& \leq 2\left\|\boldsymbol{x}_{2}\right\|\left\|P_{2}\right\| \leq 2 \mu \lambda_{\max }\left(P_{2}\right)=c_{2}
\end{aligned}
$$

- Assumption 3 is satisfied by choosing continuous functions $\alpha_{1}\left(\left\|\boldsymbol{x}_{1}\right\|\right)=\max _{\boldsymbol{z}_{2}}\left\|A_{21}\left(\boldsymbol{z}_{2}\right)\right\|\left\|\boldsymbol{x}_{1}\right\|$ and $\alpha_{2}\left(\left\|\boldsymbol{x}_{1}\right\|\right)=0$.

Since the conditions of Theorem 1 are satisfied, the cascaded system is UGAS. Furthermore, if these Lyapunov functions ensure exponential stability of the subsystems, then, based on Proposition 1, the cascaded system is also exponentially stable.
While it is true that the cascaded system is stable under the above conditions, finding a Lyapunov function valid for the cascaded system is not trivial. The construction of a crossterm in the global Lyapunov function has been proposed in [12], under the condition that the cascaded system satisfies Assumptions 2 and 3. Then, the global Lyapunov function is of the form:

$$
V_{0}\left(\boldsymbol{x}_{1}, \boldsymbol{x}_{2}\right)=V_{1}\left(\boldsymbol{x}_{1}\right)+V_{2}\left(\boldsymbol{x}_{2}\right)+\Psi\left(\boldsymbol{x}_{1}, \boldsymbol{x}_{2}\right)
$$

where $V_{1}$ and $V_{2}$ are Lyapunov functions for the systems (21) and (22), respectively.

For the case when the first subsystem is LTI, the authors of [12] proved that the cross-term exists and is continuous, and $V_{0}$ is positive definite and radially unbounded. If (21) is globally exponentially stable, the result from [12] can be extended to the system (20). The cross-term $\Psi$ is then given by:

$$
\Psi\left(\boldsymbol{x}_{1}, \boldsymbol{x}_{2}\right)=\int_{0}^{\infty} \frac{\partial V_{2}}{\partial \boldsymbol{x}_{2}}\left(\tilde{\boldsymbol{x}}_{2}(s)\right) A_{21}(\boldsymbol{z}(s)) \tilde{\boldsymbol{x}}_{1}(s) d s
$$

where $\tilde{\boldsymbol{x}}_{1}$ and $\tilde{\boldsymbol{x}}_{2}$ are the trajectories of the systems (21) and (22), respectively.

To verify the stability of the cascaded fuzzy system (19) by the Theorems 2-5 from Section II-C, additional sufficient, but by no means necessary conditions can be derived using a Lyapunov function of the form $V_{0}\left(\boldsymbol{x}_{1}, \boldsymbol{x}_{2}\right)=V_{1}\left(\boldsymbol{x}_{1}\right)+$ $V_{2}\left(\boldsymbol{x}_{2}\right)$.

For example, Theorem 2 requires a common matrix $P=$ $P^{T}>0$ so that $A_{i}^{T} P+P A_{i}<0$. Let $P=\operatorname{diag}\left[P_{1} P_{2}\right]=$ $P^{T}>0$. Then, the additional condition to those of Theorem 6 , to satisfy the requirements of Theorem 2 is the negative definiteness of $G_{i}$ for all $i=1,2, \ldots, m$ with

$$
G_{i}=\left(\begin{array}{cc}
A_{1}^{T} P_{1}+P_{1} A_{1} & A_{21}{ }^{T} P_{2} \\
P_{2} A_{21} & A_{2}{ }^{T} P_{2}+P_{2} A_{2}
\end{array}\right)_{i}
$$

In the same way, for all the above theorems, the stability conditions presented in Section II-C can be relaxed. The new conditions for Theorems 3 and 4 are presented below.

The conditions of Theorem 3 can be replaced as follows.

Theorem 7: Consider the system (19) expressed as:

$$
\dot{\boldsymbol{x}}=\sum_{i=1}^{m} w_{i}(\boldsymbol{z})\left(\begin{array}{cc}
A_{1} & 0 \\
0 & A_{2}
\end{array}\right) \boldsymbol{x}+\left(\begin{array}{c}
0 \\
A_{21}(\boldsymbol{z}) \boldsymbol{x}
\end{array}\right)
$$

This system is stable, if there exist $P_{1}=P_{1}^{T}>0, P_{2}=$ $P_{2}^{T}>0$, so that:

$$
\begin{aligned}
& A_{1 i}^{T} P_{1}+P_{1} A_{1 i}<0 \\
& A_{2 i}^{T} P_{2}+P_{2} A_{2 i}<0
\end{aligned}
$$

for all $i=1,2, \ldots, m$.

The proof is similar to that of Theorem 6 . Note that in order to satisfy all the conditions of Theorem 3 an additional condition is needed: there exists $Q=Q^{T}>0$ so that:

$$
1 \leq \frac{\lambda_{\min }(Q)}{\min _{\boldsymbol{z}}\left\|P_{2} A_{21}(\boldsymbol{z})\right\|}
$$


However, this condition is necessary only when applying Theorem 3, and is no longer needed to prove the stability of the cascaded system (20).

In order to relax the conditions of Theorem 4 let $K_{1}$ and $K_{2}$ be the number of operating and interpolation regimes for the individual subsystems, with $K_{1}^{i}$ and $K_{2}^{j}$ the index set corresponding to the local models of the subsystems active in the matching region. Then, the conditions can be expressed as:

Theorem 8: The system (15) is UGAS, if there exist matrices $P_{1}^{i}=\left(P_{1}^{i}\right)^{T}>0, P_{2}^{j}=\left(P_{2}^{j}\right)^{T}>0, H_{1}=H_{1}^{T}>0$, $H_{2}=H_{2}^{T}>0, F_{1}^{i}$, and $F_{2}^{j}, i=1,2, \ldots, K_{1}, j=$ $1,2, \ldots, K_{2}$, so that:

$$
\begin{array}{lr}
P_{1}^{i}=\left(F_{1}^{i}\right)^{T} H_{1} F_{1}^{i} & \\
P_{2}^{j}=\left(F_{2}^{j}\right)^{T} H_{2} F_{2}^{j} & \\
F_{1}^{i} \boldsymbol{x}_{1}=F_{1}^{t} \boldsymbol{x}_{1} & \forall \boldsymbol{x}_{1} \in X_{1}^{i} \cap X_{1}^{t} \\
F_{2}^{j} \boldsymbol{x}_{2}=F_{2}^{l} \boldsymbol{x}_{2} & \forall \boldsymbol{x}_{2} \in X_{2}^{j} \cap X_{2}^{l} \\
A_{1 k}^{T} P_{1}^{i}+P_{1}^{i} A_{1 k}<0 & \forall k \in K_{1}^{i} \\
A_{2 k}^{T} P_{2}^{j}+P_{2}^{j} A_{2 k}<0 & \forall k \in K_{2}^{j}
\end{array}
$$

The additional condition to satisfy Theorem 4 is:

$$
\lambda_{\min }\left(P_{1}^{j} A_{k}^{1}\right) \lambda_{\min }\left(P_{2}^{i} A_{k}^{2}\right) \leq\left\|P_{2}^{i} A_{21 k}\right\|, \quad \forall k \in K_{i}
$$

Similarly, this condition is necessary only to satisfy all the conditions of Theorem 4 and is no longer needed to prove stability of the cascaded system (20).

When applying Theorem 5, the restrictions on $G_{i i}$ and $G_{i j}$ have to be modified, which leads to conditions similar to those of Theorem 4 .

Note, that the proposed conditions are still only sufficient conditions for the stability of cascaded fuzzy systems. However, by taking the advantage of the special form of the system, i.e., studying the subsystems instead of global fuzzy system, the complexity of the associated LMI problem is reduced with respect to the presented Theorems 2-5.

\section{EXAMPLES}

In this section, the benefits of the proposed approach are demonstrated on simulation examples.

\section{A. Feasibility}

Consider the fuzzy system:

$$
\dot{\boldsymbol{x}}=\sum_{i=1}^{2} w_{i}(\boldsymbol{z}) A_{i} \boldsymbol{x}
$$

with $w_{1}(z)=w_{1}(t)=\frac{t}{\Delta t}$ and $w_{2}(t)=1-w_{1}(t)$, for all $t \in[0, \Delta t]$, i.e., the system changes in time from one local model to another.

The state matrices of the local linear models were randomly generated and are given as:

$$
A_{1}=\left(\begin{array}{cccccc}
-0.6 & 0.0 & 0.1 & 0 & 0 & 0 \\
1.2 & -1.4 & -1.8 & 0 & 0 & 0 \\
-0.3 & 1.6 & -2.8 & 0 & 0 & 0 \\
0 & 0 & 0 & -1.6 & -1.0 & -0.4 \\
0 & 0 & 0 & -0.1 & -1.2 & 0.1 \\
0 & 0 & 0 & -0.7 & 0.3 & -0.4
\end{array}\right)
$$

and

$$
A_{2}=\left(\begin{array}{cccccc}
-0.1 & 1.0 & -0.2 & 0 & 0 & 0 \\
-0.8 & -1.7 & 0.2 & 0 & 0 & 0 \\
0.3 & -1.1 & -0.9 & 0 & 0 & 0 \\
0.3 & -0.4 & 0.0 & -1.2 & 1.5 & -0.8 \\
0.1 & 0.0 & 0.9 & -0.8 & -1.0 & -0.3 \\
0.3 & -0.2 & 0.3 & 0.3 & 0.6 & -0.5
\end{array}\right)
$$

The local models are stable and simulations indicate that the system (25) is also stable. However, the LMI problem

$$
\begin{aligned}
P & >0 \\
A_{1}^{T} P+P A_{1} & <0 \\
A_{2}^{T} P+P A_{2} & <0
\end{aligned}
$$

is infeasible, so Theorems 2 and 3 cannot be applied. The stability of this system can be investigated using Theorems 4-5.

By examining the form of the system matrices, one can easily see that the system can be cascaded, with $\boldsymbol{x}_{1}=$ $\left[\begin{array}{lll}x_{1} & x_{2} & x_{3}\end{array}\right]^{T}$ and $\boldsymbol{x}_{2}=\left[\begin{array}{lll}x_{4} & x_{5} & x_{6}\end{array}\right]^{T}$.

Based on Theorem 6, the system (25) is stable if the individual subsystems are stable. As such, in order to prove the stability of the system (25), it is sufficient that the LMI problems (Theorem 6)

$$
\begin{array}{r}
P_{1}>0 \\
A_{11}^{T} P_{1}+P_{1} A_{11}<0 \\
A_{12}^{T} P_{1}+P_{1} A_{12}<0
\end{array}
$$

and

$$
\begin{aligned}
P_{2} & >0 \\
A_{12}^{T} P_{2}+P_{2} A_{12} & <0 \\
A_{22}^{T} P_{2}+P_{2} A_{22} & <0
\end{aligned}
$$

are feasible. Using Yalmip's solvesdp [20] one can easily see that it is so.

This example illustrates the main benefit of the proposed stability conditions: while the conditions imposed by conventional methods lead to an infeasible LMI system, it is still possible to prove stability of the system under study.

\section{B. Dimension reduction}

Consider the nonlinear system:

$$
\begin{aligned}
& \dot{x}_{1}=-x_{1} \\
& \dot{x}_{2}=-2 x_{2}+16 x_{1} z^{2} \sin z
\end{aligned}
$$

where $z \in[-\pi, \pi]$ is a measured variable. It can be proven that this system is globally asymptotically stable, e.g., by using the Lyapunov function $V=64 \pi^{4} x_{1}^{2}+x_{2}^{2}$.

A fuzzy approximation of this system can be obtained by linearizing the system around all $z \in$ $\{-\pi,-\pi / 2,-\pi / 4,0, \pi / 4, \pi / 2, \pi\}$. The obtained matrices 
are:

$$
\begin{aligned}
& A(-\pi)=A(0)=A(\pi)=\left(\begin{array}{cc}
-1 & 0 \\
0 & -2
\end{array}\right) \\
& A(-\pi / 2)=\left(\begin{array}{cc}
-1 & 0 \\
-4 \pi^{2} & -2
\end{array}\right) \\
& A(-\pi / 4)=\left(\begin{array}{cc}
-1 & 0 \\
-\sqrt{2} \pi^{2} / 2 & -2
\end{array}\right) \\
& A(\pi / 4)=\left(\begin{array}{cc}
-1 & 0 \\
\sqrt{2} \pi^{2} / 2 & -2
\end{array}\right) \\
& A(\pi / 2)=\left(\begin{array}{cc}
-1 & 0 \\
4 \pi^{2} & -2
\end{array}\right)
\end{aligned}
$$

i.e., there are 5 distinct local linear models. Using Theorem 2 , this means that 5 LMIs have to be solved, while in case of Theorems 4 and 5, this number is much larger (11 and 13 , respectively). However, using the proposed approach, the problem is reduced to two one-dimensional LMIs:

$$
\begin{aligned}
& -1 P_{1}+P_{1}(-1)<0 \\
& -2 P_{2}+P_{2}(-2)<0
\end{aligned}
$$

This example illustrates how, by analyzing the subsystems instead of the global fuzzy system, both the number of LMIs and their dimension can be reduced.

\section{Conclusions}

In many real-life applications, a complex process model can be decomposed into simpler, cascaded subsystems. This partitioning of a process leads to increased modularity and reduced complexity of the problem, while also making the analysis easier. In this paper, we have studied the stability of a cascaded fuzzy system, based on its individual subsystems. We have proven that the stability of such a system is determined only by the stability of the individual subsystems. Furthermore, the proposed approach relaxes the conventional stability conditions and may reduce the dimension of the problem to be solved. The benefits of studying stability based on subsystems have been demonstrated on simulation examples.

In our future research, we will investigate the theoretical conditions under which observers can be designed individually for such cascaded processes while maintaining the same performance (stability, convergence rate) as a centralized observer

\section{ACKNOWLEDGMENT}

This research is partly sponsored by Senter, Ministry of Economic Affairs of the Netherlands within the project Interactive Collaborative Information Systems (grant no. BSIK03024).

\section{REFERENCES}

[1] T. Takagi and M. Sugeno, "Fuzzy identification of systems and its applications to modeling and control," IEEE Transactions on Systems, Man, and Cybernetics, vol. 15, pp. 116-132, 1985.

[2] C. Fantuzzi and R. Rovatti, "On the approximation capabilities of the homogeneous takagi-sugeno model," in Fifth IEEE International Conference on Fuzzy Systems (FUZZ-IEEE'96), 1996, pp. 1067-1072.
[3] K. Tanaka, T. Ikeda, and H. Wang, "Fuzzy regulators and fuzzy observers: relaxed stability conditions and LMI-based designs," IEEE Transactions on Fuzzy Systems, vol. 6, pp. 250-265, 1998.

[4] M. Johansson, A. Rantzer, and K. Arzen, "Piecewise quadratic stability of fuzzy systems," IEEE Transactions on Fuzzy Systems, vol. 7, pp. 713-722, 1999.

[5] P. Bergsten, R. Palm, and D. Driankov, "Fuzzy observers," in The 10th IEEE International Conference on Fuzzy Systems, 2001, vol. 2, 2001, pp. 700-703.

[6] K. Tanaka and H. O. Wang, Fuzzy Control Systems Design and Analysis: A Linear Matrix Inequality Approach. John Wiley \& Sons, Inc., 2001.

[7] A. Loria and E. Panteley, Advanced Topics in Control Systems Theory. Springer Berlin / Heidelberg, 2005, ch. Cascaded nonlinear timevarying systems: Analysis and design, pp. 23-64.

[8] M. Arcak, D. Angeli, and E. Sontag, "A unifying integral ISS framework for stability of nonlinear cascades," SIAM J. Control Optim., vol. 40, pp. 1888-1904, 2002.

[9] E. D. Sontag, "Smooth stabilization implies coprime factorization," IEEE Transactions on Automatic Control, , vol. 34, pp. 435-443, 1989.

[10] P. Seibert and R. Suarez, "Global stabilization of nonlinear cascade systems," Systems and Control Letters, vol. 14, pp. 347-352, 1990.

[11] E. Sontag, "Remarks on stabilization and input-to-state stability," in IEEE CDC, 1989, pp. 1376-1378.

[12] M. Jankovic, R. Sepulchre, and P. Kokotovic, "Constructive Lyapunov stabilization of nonlinear cascade systems," IEEE Transactions on Automatic Control, vol. 41, pp. 1723 - 1735, 1996.

[13] A. Chaillet and A. Loria, "Necessary and sufficient conditions for uniform semiglobal practical asymptotic stability: Application to cascaded systems," Automatica, vol. 42, pp. 1899-1906, 2006.

[14] A. Bacciotti, P. Boieri, and L. Mazzi, "Linear stabilization of nonlinear cascade systems," Math. Control, Signals, Syst., vol. 6, pp. 146-165, 1993.

[15] P. Bergsten, "Observers and controllers for Takagi-Sugeno fuzzy systems," Ph.D. dissertation, Örebro University, 2001.

[16] A. Rantzer and M. Johansson, "Piecewise linear quadratic optimal control," IEEE Transactions on Automatic Control, vol. 45, pp. 629637, 2000.

[17] M. Johansson and A. Rantzer, "Computation of piecewise quadratic Lyapunov functions for hybrid systems," IEEE Transactions on Automatic Control, vol. 43, pp. 555-559, 1998.

[18] M. Johansson, "Piecewise linear control systems," Ph.D. dissertation, Department of Automatic Control, Lund Institute of Technology, 1999.

[19] W.-J. Wang and C.-H. Sun, "Relaxed stability and stabilization conditions for a TS fuzzy discrete system," Fuzzy Sets and Systems, vol. 156, pp. 208-225, 2005.

[20] J. Löfberg, " YALMIP: a toolbox for modeling and optimization in MATLAB," in Proceedings of the CACSD Conference, Taipei, Taiwan, 2004. [Online]. Available: http://control.ee.ethz.ch/ joloef/yalmip.php 